\title{
The heart exhibits right to left communication between the fibres of the muscular part of the interventricular septum
}

\author{
P.J. Kuusela \\ Private internist, Kuopio, Finland \\ [Received 28 June 2013; Accepted 30 July 2013]
}

1900 years ago Galen stated that blood seeps through the perforations in the interventricular septum. However, William Harvey, working 400 years ago, failed to find any. In this study an aqueous solution of a black dye was gently pumped by hand into the right ventricle of 20 porcine hearts. The area in the middle of the left muscular part of the interventricular septum in 13 of the hearts was bloodstained under the endocardium at the time the heart stopped beating. The same area of all 20 hearts eventually became stained black. A small amount of black dye seeped through the endocardium of 18 hearts in the middle of the left muscular part of the interventricular septum. In another 20 porcine hearts the interventricular septa were dissected after boiling. The deep pit under the anterior interventricular sulcus communicated with the right ventricle and with the middle of the left muscular part of the interventricular septum between the fibres of the muscle. The communication closed tight at the very early systole. The communication resembled that reported by Galen 1900 years ago. The communication may be the real foetal route for diastolic circulation through the muscular part of the interventricular septum from right to left. The results suggested that the anatomy, function and embryology of the African monkey, human and porcine heart are not yet fully understood. (Folia Morphol 2014; 73, 1: 42-50)

Key words: heart anatomy, heart function, heart electrophysiology, heart embryology, helical heart, septal vessel, kuuselian vessel

\section{INTRODUCTION}

Some of the first branches leaving the aorta supply the heart and brain, organs that require a high concentration of oxygen for normal development. Oxygenated blood from the umbilical circulation crosses the foramen ovale into the left atrium.

Pulmonary venous blood, which represents about $8 \%$ of total cardiac output, mixes with oxygenated blood. This blood then represents about $35 \%$ of the total foetal cardiac output. These figures are obtained from the studies in chronically instrumented close-to- -term foetal lambs. The more recent Doppler velocity evaluation suggests in human right ventricular (RV) cardiac output dominance $57 \%$. The blood pressures in the main pulmonary trunk and RV are $1-2 \mathrm{~mm} \mathrm{Hg}$ higher than those in the aorta and left ventricle (LV) [9].

The sinoatrial node initially activates the right atrium, followed by the activation of the left atrium 6 to 10 ms later [7]. In most cases the left His bundle has 3 fascicles: anterior, posterior and septal. The activation of the middle third of the septum occurs 
5 ms before that of the postero-inferior and antero-superior regions [14]. At 5 to $10 \mathrm{~ms}$ after the onset of ventricular activation the wave of activation envelops the LV and RV walls and the remainder of the septum: the latter is completely activated at $12 \mathrm{~ms}$ [7].

Abnormal fourth heart sounds originate in either the LV or the RV and disappear when coordinated atrial contraction ceases, as in atrial fibrillation. The abnormal fourth heart sound is common in hypertrophy of systemic hypertension and in ischaemic heart disease, because the atrial 'booster pump' is needed to assist the relatively stiff ischaemic ventricle [15]. The adult right atrial systolic and RV end-diastolic pressures are 1-2 to $7 \mathrm{~mm} \mathrm{Hg}$. The LV end-diastolic pressure is higher at 2-12 $\mathrm{mm} \mathrm{Hg}$. RV ischaemia and diminished compliance result in elevated RV end-diastolic pressure [6].

Francisco Torrent-Guasp dissected hearts after boiling. The LV myocardial band of the heart has a helical configuration formed by the descendant segment and the ascendant segment, which lie over the anterior wall of the LV. At the apex, the laminar organisation of the muscle fibre groups twists subendocardially around a tunnel and the subepicardial fibres become subendocardial around this tunnel. The basal LV has the same helical trajectory. The fibres of the muscle pass from the outside to the inside of the ventricular wall without inserting into the mitral ring. The helical course of the laminar trajectories under the endocardium runs in the opposite direction to those at the apex. The heart widens at the end of the diastole by ventricular band relaxation [17].

Small cardiac veins (Thebesian vessels) are the minimal openings into all cavities of the heart. Numerous small RV veins up to $0.5 \mathrm{~mm}$ diameter open into the RV [18]. The small RV veins system can be displayed by injecting the material from either the sinus coronarius or the RV. Very slender small RV veins, emptying into the RV, are seen in the proximal half of the septum. At the mid-height of the interventricular septum (IVS) there is an area of venous bifurcation: the rami septi ventrales are directed towards the great cardiac vein, the rami septi dorsales, again, towards the posterior interventricular sulcus [1].

In the first stage of the formation of the foetal muscular part of the IVS, masses of actively growing tissue fuse together to bridge and divide the arterial pole of the heart into the pulmonary and aortic outflow tracts. In the second stage the expansion of a narrow ridge of compressed trabeculae forms be- tween the ventricular bodies. The septum is formed by compaction of trabeculations. As the expanding portions continue to grow, their medial walls approach and fuse, forming the septum. The interventricular foramen is closed by the membranous part of the IVS at the end of the $7^{\text {th }}$ week [5]. Ben-Shachar et al. [4] use scanning electron microscopy combined with critical point drying of the tissue. They find the smooth endocardial-lined channels to be oriented left-right and craniocaudal directions in the middle of the muscular part of the IVS of the stage 30 chick embryo heart. The trabecular endocardial surface becomes apposed and forms into channels through coalescence of the trabecular sheets. They speculate that highly organised ventricular trabeculations may regulate the systolic and diastolic functions of the primitive ventricle and that the muscular ventricular septal defects (VSD) are due to continued patency of the channels in the muscular part of the IVS of the fully developed heart [4].

A congenital VSD of the heart may appear in any part of the septum [3]. A single VSD of the left central muscular part of the IVS may present with numerous sieve-like perforations through the right muscular part of the IVS formed from the medial wall of the foetal RV [2]. A small hole in the membranous part of the IVS becomes a hidden communication through the membranous part of the IVS as the aneurysm of the membranous part of the IVS grows over the hole on the right side after birth [10,13].

Galen was born in 130 A.D. and studied medicine for 11 years in Egypt [11]. He boiled and dissected hearts. He also dissected living animals (vivisection), particularly African monkeys and vivisected the LV. He saw the blood flows out of the coronary veins and arteries of the anterior interventricular sulcus and right to left through the central muscular part of the IVS. Galen stated they could be found exactly by carefully dissecting a freshly-killed animal. The force behind arterial pulsation has its origin in the heart. Liquid from the stomach is absorbed into the veins. Galen stated that blood passes from the RV through the parenchyma of the lungs into the LV and that the lungs convey pneuma (oxygen) into the blood [16]. Arteries and veins have common openings in the body and between the ventricles of the heart for proper nourishment. The liver is nourished almost entirely by the heaviest venous blood and the brain by the lightest (porous) blood. A considerable quantity of 
blood already elaborated in the veins is taken over through the central muscular part of the IVS and its perforations into the LV and into the arteries. The deep pits in the IVS of the heart are formed for the purpose of communication. The organs need different kinds of useful blood to remain viable [12]. William Harvey boiled and dissected hearts. He did not vivisect the LV of the heart. He stated that all fibres in the septum of a boiled heart are circular as in a sphincter. He demonstrated from the structure of the heart and by observing beating hearts that blood passes from the RV through the lungs into the LV. Harvey ligated the pulmonary artery and fastened a small tube through the vena cava into the RV of the human heart. He forced warm water at considerable pressure into the RV but not a drop of water or blood escaped through the gap into the LV of the heart. Using the same method he demonstrated that water passes from the pulmonary artery through the lungs into the LV of the heart [8]. Harvey published "De Motu Cordis" in 1628. His views on blood circulation became generally accepted within 30 years. Harvey wondered why Galen failed to grasp the correct idea of the circulation [11].

The present author noticed a flow from right to left through the IVS of the heart. Cardiologists claimed this was an artefact of the ultrasound imaging system. Galen presumably vivisected the still living LV of the heart and saw that blood was taken over through the IVS. In the present study an aqueous solution of a black dye was squeezed gently with the fingers through the pulmonary artery into the closed RV and, consistent with Galen's observations, the author saw dye seeping through the homogeneous central muscular part of the IVS right to left. This communication was preliminarily identified by dissecting fresh pig hearts. The author also dissected the IVS of the pig hearts after boiling and identified the communication completely.

\section{MATERIALS AND METHODS}

Fourty pig hearts were regular pig hearts destined for the food industry. All the pigs were aged 6 months and weighed about $50 \mathrm{~kg}$ to $55 \mathrm{~kg}$. The sex was unknown and not selected. The pigs were anaesthetised using $80 \%$ to $85 \%$ carbon dioxide inhalation and a blade was inserted into the common carotid artery. The pigs were relaxed and blood pressure was normal at the time of slaughter. The hearts had no anatomical malformations (Personal communication by Irmeli Sippola [a VET] working for the Government of Finland at the Atria Company that complies with the laws of Finland and directives of European Union). The author employed 2 methods, designated A and B.

\section{Method A}

The vena cava and the right atrioventricular orifice of 20 porcine hearts were ligated. The LV wall left to the anterior interventricular sulcus and apical to the coronary sulcus was incised. The wall of the LV was deflected to the left. A plastic bottle containing an aqueous solution of black dye was squeezed gently and repeatedly with the fingers through the pulmonary artery into the RV, thus mimicking the right atrial cycle. The IVS was inspected visually to observe whether any of the black solution was seeping through. Next, the RV was dissected in the same manner as the LV. The inside corner between the septum and the right anterior ventricular wall was dissected in order to inspect the deep pits under the anterior interventricular sulcus. The septum between the deep pits and the central muscular part of the IVS was dissected in order to find the communication and any black dye between the deep pit and the left central muscular part of the IVS. The author suspected that dye may flow into the tissue of the heart through the coronary veins. Ten porcine hearts had the coronary veins patent and 10 porcine hearts also had the coronary veins ligated near the coronary sinus. Time between the slaughter and the experiment was 2-7 days. Twenty warm hearts in a plastic bag were cooled in a refrigerator. The hearts stopped beating at the systole and relaxed again after 2 days.

\section{Method B}

The walls of both ventricles of the other 20 pig hearts were removed. The IVS of the pig hearts was kept in boiling water for $2 \mathrm{~h}$. The left endocardium was removed (Fig. 1). The septum was incised anterior to posterior through 2 openings of the communication in the middle of the left muscular part of the IVS (Fig. 2). The layers of the fibres of the muscle were carefully removed as far as the outer layer of the left muscular part of the IVS and the communication anterior to the openings of the communication were visible (Figs. 3, 4). 


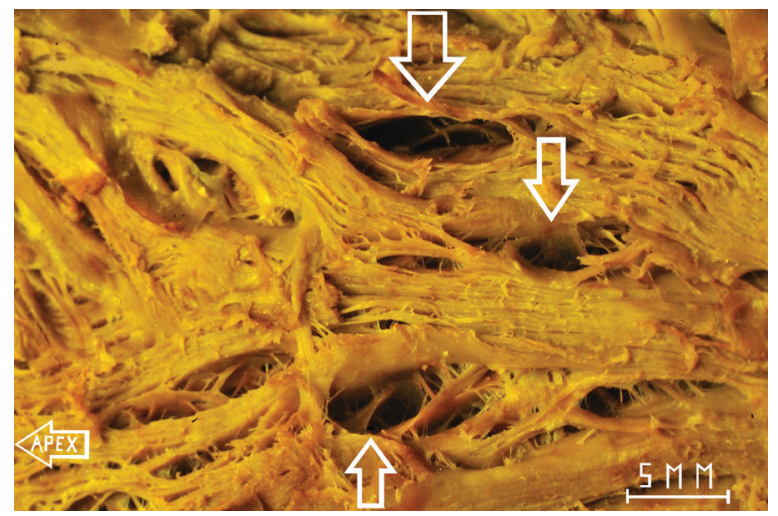

Figure 1. The endocardium of the left muscular part of the interventricular septum was removed. The septum was slightly bent. Three empty openings of the septal vessels (kuuselian vessels) from right to left through the left muscular part of the interventricular septum were visible (arrows).

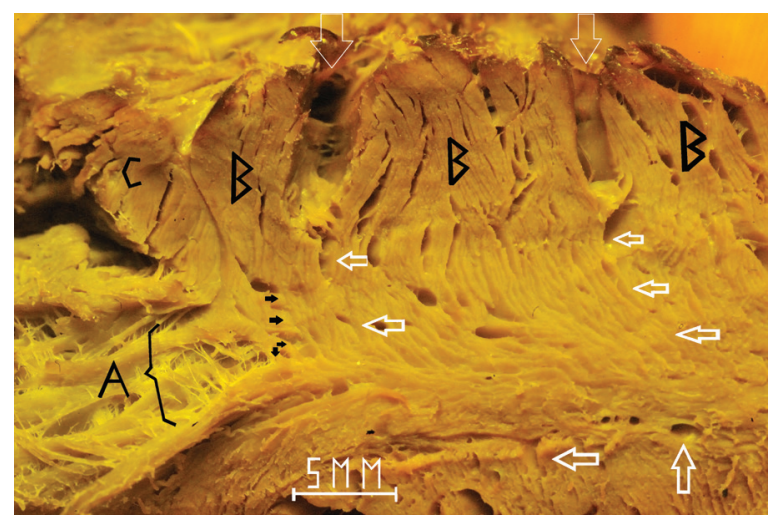

Figure 2. The septum had the transversal dissection through 2 openings (upper arrows). The narrow septal vessels (kuuselian vessels) between the sphincters curved posteriorly towards the right muscular part of the interventricular septum (right white arrows). The muscle fibre (A) had 4 insertions into the functional sphincter of the septal vessel (kuuselian vessel) in the outer layer of the left central muscular part of the interventricular septum (left black arrows). The septal vessel (kuuselian vessel) was located between the fibres of the muscle that formed the $1 \mathrm{~cm}$ wide band anteriorly $(A)$. The muscle of the anterior wall of the left ventricle (LV) had its origin through the left central muscular part of the interventricular septum (B). The band of muscle $A$ and $B$ formed the part of the right half of the ascendant segment of the ventricular myocardial band over the subepicardial anterior wall of the LV. The muscle of the anterior wall of the LV might have spread out the sphincter of the septal vessel (kuuselian vessel) through the left muscular part of the interventricular septum, as the LV of the foetal heart widened at the diastole. Small right ventricular veins (Thebesian veins) did not communicate with left muscular part of the interventricular septum (lower arrows). C was a part of the descendant segment of the ventricular myocardial band inside the muscular band formed from muscle $A$ and $B$.

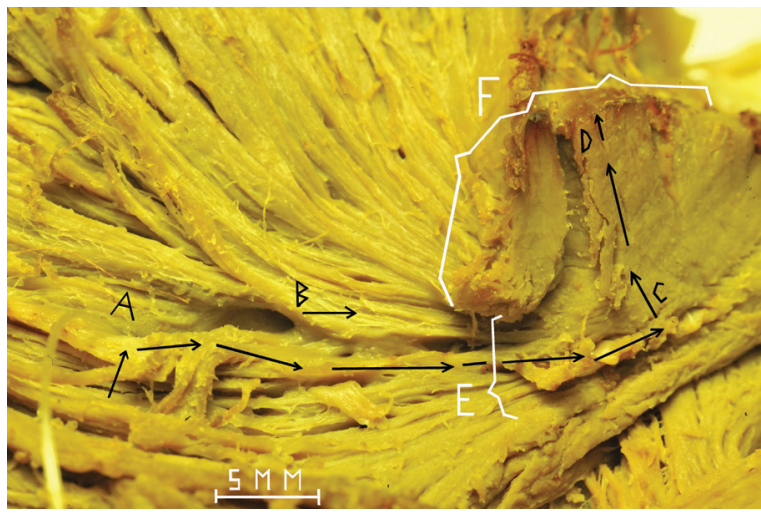

Figure 3. The septal vessel (kuuselian vessel) from right to left through the muscular part of the interventricular septum of the heart was visible between the fibres of the outer muscular part of the left interventricular septum of the heart (black arrow). The line passed through the deep pit to the right ventricle (A). The septal vessel (kuuselian vessel) had a branch (B). The septal vessel (kuuselian vessel) laid at an angle of more than 90 degrees towards the left ventricle (LV) (C). The opening of the septal vessel (kuuselian vessel) into the LV was located in the middle of the left muscular part of the interventricular septum (D). The $1 \mathrm{~cm}$ wide band of muscle fibres had the insertion into the sphincter of the septal vessel (kuuselian vessel) partially removed $(E)$. The band of muscle fibres $E$ and $F$ extended over the anterior wall of the LV. The band of muscle $E$ and $F$ formed a part of the right half of the ascendant segment of the ventricular myocardial band over the anterior wall of the LV.

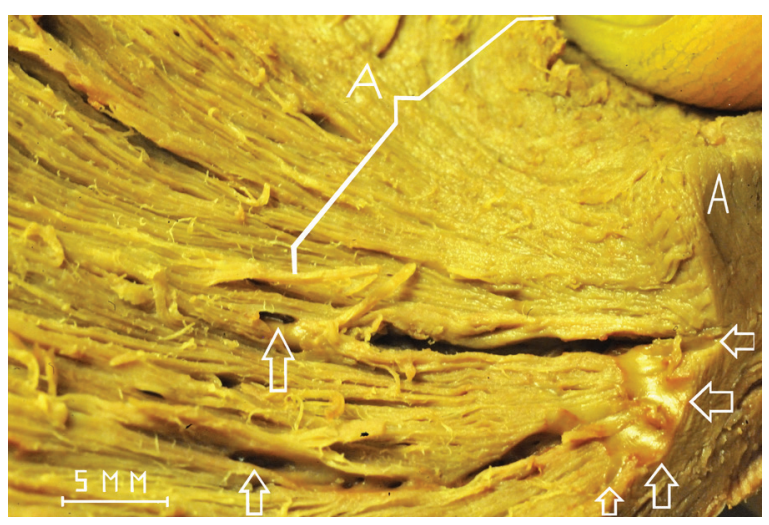

Figure 4. Two septal vessels (kuuselian vessels) were located between the fibres of the muscle of the outer layer of the left muscular part of the interventricular septum. The septal vessels (kuuselian vessels) were to the right ventricle of the heart (left arrows). The septal vessels (kuuselian vessels) were right to the removed sphincter (right arrows). The fibres of the left ventricle between the aortic base and middle of the anterior interventricular sulcus became subendocardial and formed a laminar trajectory and the sphincter in the middle of the left muscular part of the interventricular septum (A).

\section{RESULTS}

\section{General description}

Method A. The deep pit had a large communication with the RV. The communication had an opening in the deep pit towards the central muscular part of 


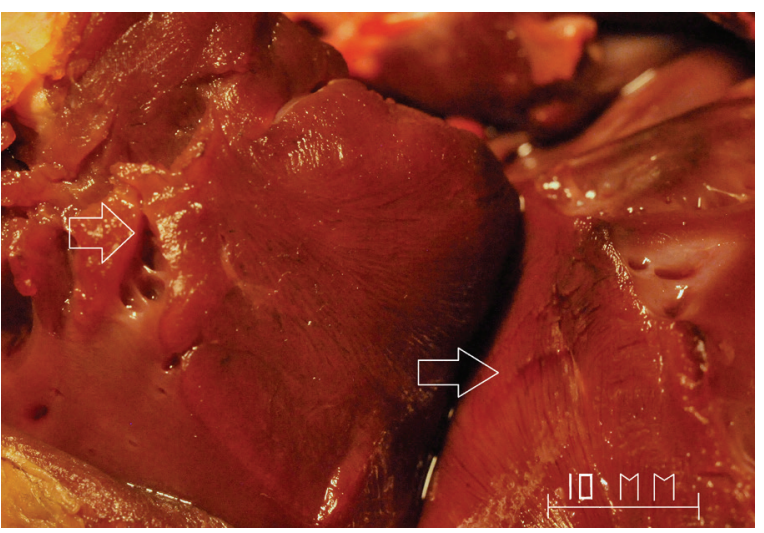

Figure 5. The septum had the transversal dissection. The deep pit had a wide communication with the right ventricle (RV). The deep pit passed through the right interventricular septum formed from the medial wall of the expanding foetal RV (left arrow). Black dye stained the septal vessel (kuuselian vessel) left of the sphincter and the surrounding tissue through the left central muscular part of the interventricular septum formed from the medial wall of the expanding foetal left ventricle (right arrow).

the IVS. The endocardium in the middle of the left muscular part of the IVS of 13 hearts was bloodstained. The same area became black when dye was squeezed through the pulmonary artery into the RV of all 20 hearts. A small amount of dye seeped into the LV of 18 hearts (Fig. 5).

Method B. The communication with the sphincter was visible right to left between the fibres of the muscular part of the IVS on dissection of the other 20 pig hearts after boiling (Fig. 3). The term 'septal vessel' (kuuselian vessel) referred to the communication between the fibres of the muscle right to left through the muscular part of the IVS of the heart.

\section{Description in detail}

Method A. There was a deep pit $4.5 \mathrm{~cm}$ caudally to the aortic valve and $2 \mathrm{~cm}$ to the left central muscular part of the IVS through the right IVS formed from the medial wall of the expanding foetal RV communicating to the RV. The deep pit had an opening of the septal vessel (kuuselian vessel) $1 \mathrm{~mm}$ or less in diameter between the right and left muscular part of the IVS. In 19 of the 20 hearts the septal vessel (kuuselian vessel) was visible between the fibres of the muscle most commonly located $1 \mathrm{~cm}(5-20 \mathrm{~mm})$ towards the black central muscular part of the IVS (Figs. 5-8). A small amount of black dye was present

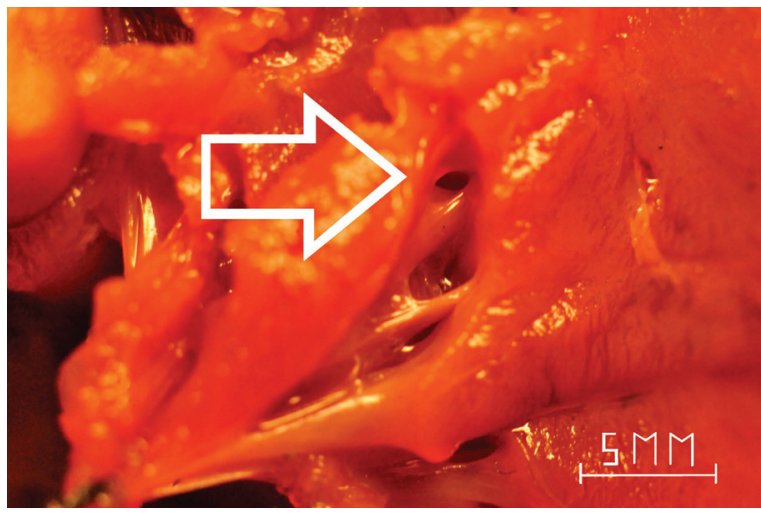

Figure 6. The opening of the septal vessel (kuuselian vessel) was located in the deep pit in the middle of the muscular part of the interventricular septum and under the anterior interventricular sulcus (arrow). The septal vessel (kuuselian vessel) had a diameter of $1 \mathrm{~mm}$ or less. The wall of the septal vessel (kuuselian vessel) was a transparent wall of muscle fibre. The septal vessel (kuuselian vessel) was formed between the medial walls of the expanding foetal right and left ventricles.

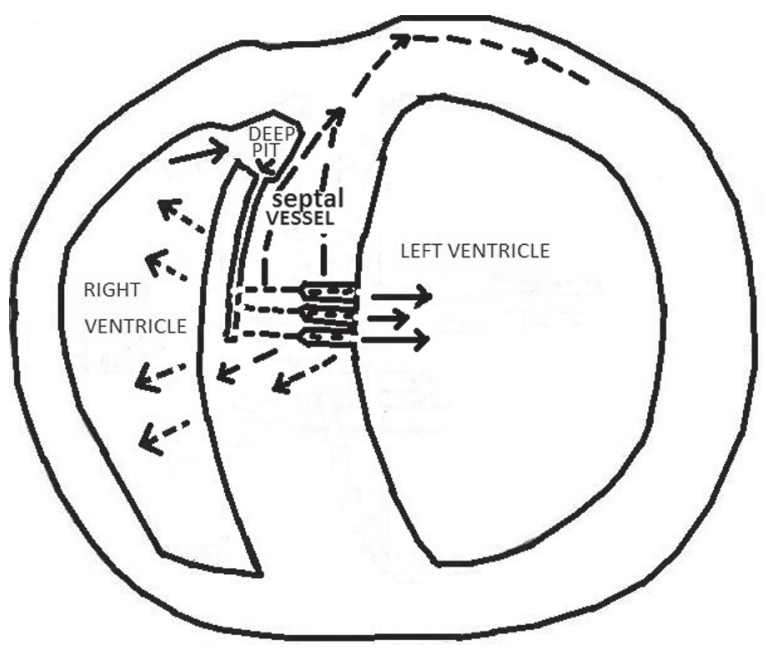

Figure 7. Right to left communication through the interventricular septum (IVS) of the heart: the deep pit was located under the anterior interventricular sulcus. The deep pit passed through the right IVS formed from the medial wall of the expanding foetal right ventricle (RV), which was moving towards the RV at the diastole (broken arrows). The septal vessel (kuuselian vessel) was formed between the expanding medial walls of the foetal RV and left ventricle (LV) towards the central muscular part of the IVS and into the LV. The ascendant segment of the ventricular myocardial band over the anterior wall of the LV had a $1 \mathrm{~cm}$ wide band of insertions into the sphincter of the septal vessel (kuuselian vessel) and the laminar trajectory in the left central muscular part of the LV. The band of muscle may have spread out the septal vessel (kuuselian vessel) through the left central muscular part of the IVS at the foetal diastole (long broken arrow). Black dye seeped between the fibres of the muscle under the endocardium through the muscular IVS formed from the medial wall of the expanding foetal LV, which was moving towards the RV at the diastole (broken arrows). Black dye seeped through the endocardium of the LV (right arrows). 


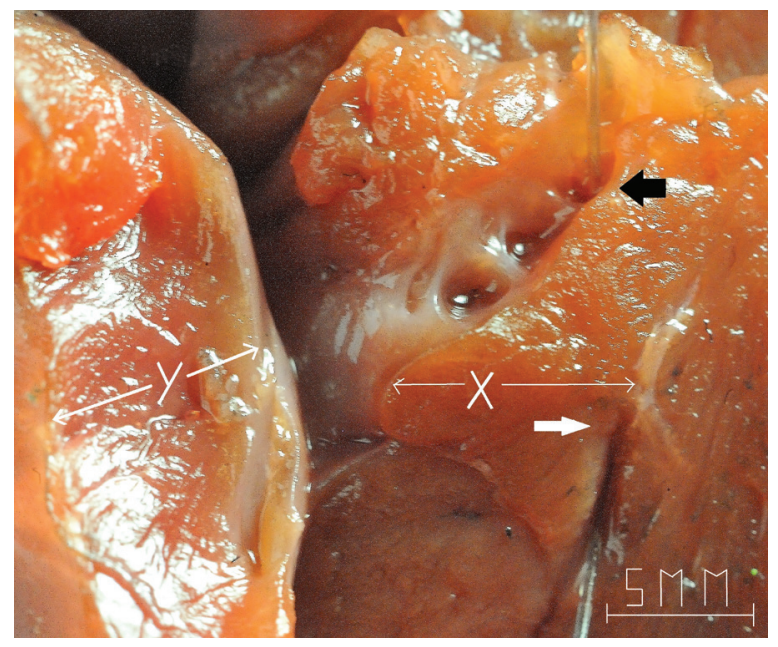

Figure 8. The $0.3 \mathrm{~mm}$ diameter line went without resistance $7 \mathrm{~mm}$ into the septal vessel (kuuselian vessel) through the deep pit (black arrow). The line perforated the fragile septal vessel (kuuselian vessel) and came out between the muscular interventricular septum formed from the expanding foetal right and left ventricles (white arrow); $Y$ — the wall of the right ventricle; $X$ — the muscular interventricular septum formed from the medial wall of the expanding foetal right ventricle.

inside the septal vessel (kuuselian vessel) of 5 hearts, most commonly $1 \mathrm{~cm}$ from the deep pit.

The IVS of 13 hearts was bloodstained under the endocardium in the middle of the left muscular part of the IVS located 4-6 cm from the aortic valve. The hearts of group 1 (Table 1) and group 2 (Table 2) had 7 and 6 interventricular septa, respectively, with a bloodstained area under the left endocardium ranging from $3 \mathrm{~mm}$ in diameter to an area of $10 \times 13 \mathrm{~mm}$.
The IVS of 7 hearts was not bloodstained under the endocardium, because the blood ran off the communication completely as these 7 hearts stopped beating at the systole. The black dye stained the same area in all 20 hearts. The area stained varied from $5 \times 5 \mathrm{~mm}$ to $6 \times 3 \mathrm{~cm}$. The most common size was $2 \times 2 \mathrm{~cm}$. A small amount of black dye seeped through the endocardium into the LV in a total of 9 septa of both groups.

The dye made irregular lines towards the endocardium between the fibres of the muscle through the left central muscular part of the IVS formed from the expanding medial wall of the foetal LV. The homogeneous left central muscular part of the IVS had no canals and the dye stained the fibres of the muscle through the septum. The stripe filled with tightly packed black dye was under the endocardium like the endocardial-lined channels presented by Ben-Shachar et al. [4]. The black dye also stained the route through the endocardium slightly black (Figs. 5, 9).

Twenty-three drops of the black dye seeped through the first 10 septa and 15 drops through the last and older 10 septa. No black dye seeped through the last and oldest left endocardium of the septum for about a week in both groups. The communication through the endocardium lost patency as the hearts became older, probably due to necrosis or degeneration, which did not produce artificial channels through the tissue. Muscular autolysis made no artificial channels (Tables 1, 2).

The coronary veins of 10 hearts in group 1 were patent (Table 1), while the coronary veins of 10 he-

Table 1. Hearts 1-10 had the coronary veins patent: the presence and size of the bloodstained area $(A, m m)$ and the dye-stained area $(B, c m)$ in the middle of the left muscular part of the left ventricle is shown; the number of points with drops of black dye seeping through the muscular part of the ventricular septum is shown (C); the communication (septal vessel) length is shown (D, cm); dye staining inside the former $(E+$ or - ); the posterior papillary muscle was black $(F+$ or - ); the posterior wall of the left ventricle had the black area $1.5 \mathrm{~cm},+$ or $-(\mathrm{G})$

\begin{tabular}{lccccccc}
\hline Heart no. & A & B & C & D & E & F & G \\
\hline 1 & 0 & $2 \times 3$ & 3 & 2 & - & - & - \\
2 & $3 \times 3$ & 0.3 & 2 & $2 \times 1$ & + & - & - \\
3 & $10 \times 10$ & $3 \times 3$ & 2 & 1 & + & + & + \\
4 & $5 \times 15$ & $3 \times 6$ & 2 & 2 & - & + & + \\
5 & $5 \times 8$ & $1.5 \times 2$ & 3 & 0.5 & + & - & - \\
6 & 0 & $2 \times 4$ & 3 & 1 & - & + & + \\
7 & $10 \times 13$ & $5 \times 5.5$ & 1 & 2 & + & - & - \\
8 & 0 & $1 \times 1.5$ & 1 & 0.5 & - & + & - \\
9 & 5 & $2 \times 2$ & 3 & 1 & - & + & - \\
10 & 5 & $3 \times 4$ & 0 & 1 & - & + & + \\
\hline
\end{tabular}




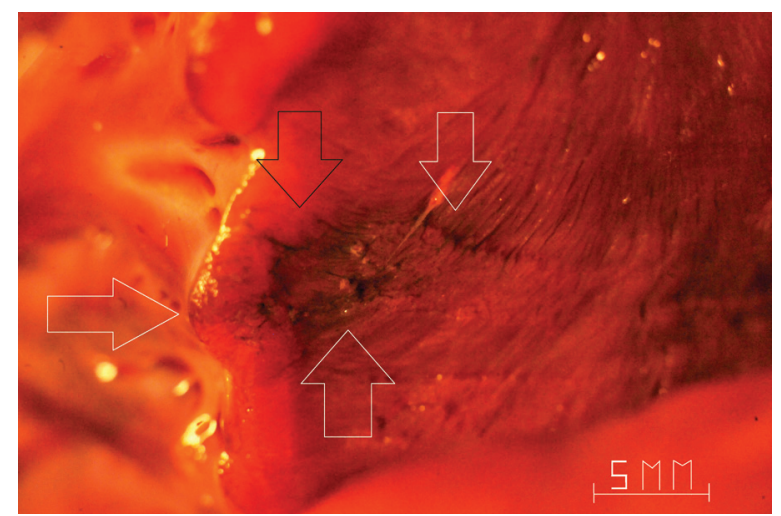

Figure 9. Black dye seeped under the endocardium and made the irregular lines between the fibres of the muscle in the middle of the left muscular part of the interventricular septum formed from the medial wall of the expanding foetal left ventricle (LV) (right arrows). The black stripe filled with tightly packed black dye resembles the endocardial channel found by Ben-Shachar et al. in 1985 [4] (black arrow). The black dye stained the route through the endocardium of the LV (left arrow).

arts in group 2 were ligated (Table 2). Four of the hearts in group 1 exhibited black staining of the posterior endocardium of the LV close to the right atrium and the sinus coronarius, most common $10 \times 15 \mathrm{~mm}$ in size, whereas none of 10 hearts in group 2 showed any staining. The posterior papillary muscle showed some black staining in 6 hearts in group 1 and in 1 heart in group 2 . One coronary vein ligation in group 2 might not have been complete. The other results were similar for both groups. The right muscular part of the IVSs did not exhibit black staining. Black dye was not forced through the small
RV veins (Thebesian vessels) (Fig. 2) and it did not reach the central muscular part of the IVS through the coronary veins.

Method B. The interventricular septa of 20 pig hearts were dissected after boiling in water for $2 \mathrm{~h}$ (Table 3). Boiling caused the IVS to shrink. The fibres of the muscle of the septal vessel (kuuselian vessel) opposite to each other dissociated as the surrounding tissue shrank. The tissue also lost fat as a result of boiling. The RV had an opening into the septal vessel (kuuselian vessel) through the deep pit under the anterior interventricular sulcus in the middle of the IVS. The septal vessel (kuuselian vessel) reached the left central muscular part of the IVS between the muscular fibres (Figs. 3, 4). The fibres of the muscle surrounding the septal vessel (kuuselian vessel) extended over the anterior wall of the LV. The fibres of the muscle surrounding the septal vessel (kuuselian vessel) had 90 degrees grossing of the band of the muscle at the outer layer of the left central muscular part of the IVS by a $1 \mathrm{~cm}$ wide band of insertions (Fig. 2). The outer left central muscular part of the IVS was formed from the dense laminar trajectory that was the sphincter of the septal vessel (kuuselian vessel). The septal vessel (kuuselian vessel) had an angle of more than 90 degrees through the sphincter into the LV. Under the endocardium of the left central muscular part of the IVS the septal vessel (kuuselian vessel) had 2 or 3 oval $2 \times 5 \mathrm{~mm}$ openings. The depth of the openings was half of the left central muscular part of the IVS (Figs. 1-4).

Table 2. Hearts 11-20 had the coronary veins ligated: the presence and size of the bloodstained area $(A, \mathrm{~mm})$ and the dye-stained area $(B, c m)$ in the middle of the left muscular part of the left ventricle is shown; the number of points with drops of black dye seeping through the muscular part of the ventricular septum is shown $(C)$; the communication (septal vessel) length is shown $(D, c m)$; dye staining inside the former $(E+$ or - ); the posterior papillary muscle was black $(F+$ or -$)$; the posterior wall of the left ventricle had the black area $1.5 \mathrm{~cm}(\mathrm{G}+$ or -$)$

\begin{tabular}{lccccccc}
\hline Heart no. & $\mathbf{A}$ & $\mathbf{B}$ & $\mathbf{C}$ & $\mathbf{D}$ & $\mathbf{E}$ & $\mathbf{F}$ & $\mathbf{G}$ \\
\hline 11 & $5 \times 8$ & $3 \times 3$ & 2 & 2 & - & - & - \\
12 & $10 \times 20$ & $1.5 \times 2$ & 2 & 2 & + & - & - \\
13 & $2 \times(5 \times 5)$ & $2 \times 3$ & 4 & $2 \times 1$ & - & - & - \\
14 & $5 \times 5$ & $1.5 \times 3$ & 2 & 1 & - & - & - \\
15 & 0 & $5 \times 5$ & 1 & 0.5 & - & - & - \\
16 & 0 & $2 \times 3$ & 2 & 1 & - & - & - \\
17 & 0 & $2 \times(1 \times 1)$ & 3 & 2 & - & - & - \\
18 & $8 \times 10$ & $1.5 \times 3$ & 1 & 1 & - & - & - \\
19 & $20 \times 20$ & $1 \times 1.5$ & 1 & 0.5 & - & - & - \\
20 & 0 & $1 \times 4$ & 0 & 0 & - & - & - \\
\hline
\end{tabular}


Table 3. The interventricular septa of hearts 1-20 were kept in boiling water for $2 \mathrm{~h}$ : the number of openings of the communication (septal vessel) in the middle of the muscular part of the interventricular septum is shown $(A)$; the number of $0.5-3 \mathrm{~mm}$ wide and $5-17 \mathrm{~mm}$ long communication is shown (septal vessel) (B); the number of openings between the right ventricle and the communication is shown (septal vessel) (C); the communication (septal vessel) right to the sphincter pointed to mild functional activity by dilatation and tone of dark colour (D)

\begin{tabular}{|c|c|c|c|c|}
\hline Heart no. & $A$ & B & C & D \\
\hline 1 & 3 & 1 & 1 & \\
\hline 2 & 3 & 2 & 2 & \\
\hline 3 & 3 & 1 & 1 & \\
\hline 4 & 3 & 1 & 1 & \\
\hline 5 & 3 & 1 & 1 & $x$ \\
\hline 6 & 2 & 1 & 1 & \\
\hline 7 & 2 & 1 & 1 & $x$ \\
\hline 8 & 3 & 1 & 1 & $x$ \\
\hline 9 & 2 & 1 & 1 & \\
\hline 10 & 2 & 2 & 2 & $x$ \\
\hline 11 & 3 & 1 & 1 & \\
\hline 12 & 3 & 2 & 2 & \\
\hline 13 & 2 & 1 & 1 & \\
\hline 14 & 3 & 1 & 1 & \\
\hline 15 & 3 & 1 & 1 & \\
\hline 16 & 3 & 1 & 1 & \\
\hline 17 & 3 & 2 & 2 & \\
\hline 18 & 3 & 1 & 1 & \\
\hline 19 & 3 & 1 & 1 & \\
\hline 20 & 3 & 1 & 1 & \\
\hline
\end{tabular}

The sphincter in the middle of the septum was formed from the fibres of the anterior wall of the LV. The fibres of the LV between the aortic base and the middle of the anterior interventricular sulcus extended to the middle of the left muscular part of the IVS and became subendocardial, forming the laminar trajectory and the sphincter in the middle of the left muscular part of the IVS (Figs. 3, 4). The sphincter communicated with the apical and helical muscular trajectory close to the apex (Fig. 1).

\section{DISCUSSION}

The septal vessel (kuuselian vessel) was surrounded by the sphincter in the middle of the left muscular part of the IVS in agreement with William Harvey. He stated that all the fibres in the septum of a boiled heart are circular as in a sphincter [8]. Activation of the middle third of the septum occurs $5 \mathrm{~ms}$ before that of the postero-inferior and antero-superior regions [14]. The septal fascicle of the left His system closed the sphincter as the systole of the LV was beginning. The left to right communication did not result as the earliest $L V$ activation closed the sphincter of the septal vessel (kuuselian vessel) at the very early systole. Venous bifurcation area of the central muscular part of the IVS was also concordant with the sphincter of the septal vessel (kuuselian vessel) [1]. The results were also concordant with a single muscular VSD of the left central muscular part of the IVS, with numerous sieve-like perforations through the right muscular part of the IVS formed from the medial wall of the foetal RV [2]. Agenesis of the sphincter of the septal vessel (kuuselian vessel) in the middle of the left IVS of the heart was the cause of the VSD. The septal vessel (kuuselian vessel) resembled that reported by Galen 1900 years ago $[12,16]$.

The sphincter of the septal vessel (kuuselian vessel) was feasible to be patent by relaxing and widening the LV at the foetal diastole. These findings were in agreement with Francisco Torrent-Guasp's model of the helical heart, although he did not report the laminar trajectory to be in the middle of the left muscular part of the IVS [17]. The septal vessel (kuuselian vessel) may have become patent right to left by the diastolic widening of the foetal heart for the diastolic blood pressure, which is higher in the RV than in the LV. Pulmonary venous blood, which represents about $8 \%$ of total cardiac output, mixes with oxygenated blood in the left atrium [9]. The sinoatrial node initially activates the right atrium [7]. Oxygenated blood from the umbilical circulation might have reached the outflow tract of the LV and the arteries of the heart and brain through the central muscular part of the IVS. The septal vessel (kuuselian vessel) was the novel structure for the circulation right to left through the muscular part of the IVS of the foetal heart.

Black dye solution seeped right to left through the IVS of all 20 pig hearts. Shrinking of the surrounding tissue by boiling was essential to dissociate the fibres of the muscle around the septal vessel (kuuselian vessel) of the IVS of the other 20 pig hearts. Boiling for $1 \mathrm{~h}$ was not enough. The author inspected the septal vessel (kuuselian vessel) of the fresh hearts and after boiling in water for $2 \mathrm{~h}$. In both groups the septal 
vessel (kuuselian vessel) was surrounded by fibres of the muscle. Boiling did not change the appearance of the septal vessel (kuuselian vessel). The septal vessel (kuuselian vessel) was not a canal or channel or blood vessel, but a slit between the fibres of the muscle. The septal vessel (kuuselian vessel) was feasible to appear at the diastole and close tight at the systole.

Black dye seeped through the first 10 septa more abundantly than through the last and older 10 septa. The author conducted experiments on 3 fresh, still warm hearts at the Butcher's Broom. However, the hearts stopped beating at the systole. No black dye penetrated into the IVS, although a drop of bloody fluid seeped out of the homogeneous left central muscular part of the IVS of all 3 hearts, in agreement with 13 bloodstained areas presented by the results. The results suggest that black dye and blood ran off the communication. For the photographs, the hearts were cooled by $-16^{\circ} \mathrm{C}$. The hearts had not relaxed after 2 days in the refrigerator. They were relaxed by passing lukewarm water into and out of the RV from a plastic bottle. Forcing water at high pressure from the bottle also closed the septal vessel (kuuselian vessel) and ruined the method.

The pathophysiological question that remained to be answered was: Was it possible to image by angiography the small amount of poorly oxygenated blood flowing through the central muscular part of the IVS from the RV into the LV of the adult heart due to disease and generate the fourth heart sound at the right atrial filling phase? Hypoxia promotes atherosclerosis of heart and brain arteries, heart insufficiency and senile dementia.

The end-diastolic relaxation and widening of the foetal heart at the right atrial filling phase is a fascinating phenomenon, but what was its function? The results suggested that we do not yet fully understand the anatomy, function and embryology of the porcine, African monkey and human heart.

\section{ACKNOWLEDGEMENTS}

The author would like to thank Raimo Leppänen for his assistance with computer problems, Tero Heiskanen for his help with the information and Tero Rasanen for his help with photography.

The author bought the hearts from the butcher's of Atria Company in Finland. Atria Company complies with the laws of Finland and the directives of European Union.

\section{REFERENCES}

1. Aho A (1950) On the venous network of the human heart and its arteriovenous anastomoses. Ann Med Exp Biol Fenn, 28 (suppl. 1): 1-90.

2. Asou T (2011) Surgical management of muscular trabecular ventricular septal defects. Gen Thorac Cardiovasc Surg, 59:723-729.

3. Baker EJ, Leung MP, Anderson RH, Fischer DR, Zuberbuhler JR (1988) The cross sectional anatomy of ventricular septal defects: a reappraisal. Br Heart J, 59: 339-351.

4. Ben-Shachar G, Arcilla RA, Lucas RV, Manasek FJ (1985) Ventricular trabeculations in the chick embryo heart and their contribution to ventricular and muscular septal development. Circ Res, 57: 759-766.

5. Clark EB, Van Mierop L HS (1989) Development of the cardiovascular system. In: Adams FH, Emmanouilides GC, Riemenschneider TA eds. Heart disease in infants, children, and adolescents. $4^{\text {th }}$ Ed. Williams \& Wilkins, Baltimore, pp. 2-10.

6. Davidson CJ, Fishman RF, Bonow RO (1997) Cardiac catheterization. In: Braunwald E ed. Heart disease. $5^{\text {th }}$ Ed. WB Saunders, Philadelphia, pp.188-191.

7. Fisch C (1997) Electrocardiography. In: Braunwald E ed. Heart disease. $5^{\text {th }}$ Ed. WB Saunders, Philadelphia, pp. 108-111.

8. Franklin JK (1963) Harvey: The circulation of the blood and other writings. JM. Dent \& Sons Ltd, London, pp. 1-236, 107, 183-184.

9. Heymann MA (1989) Fetal and neonatal circulations. In: Adams FH, Emmanouilides GC, Riemenschneider TA. Heart disease in infants, children, and adolescents. $4^{\text {th }} \mathrm{Ed}$. Williams \& Wilkins, Baltimore, pp. 24-32.

10. Jain AC, Rosenthal R (1967) Aneurysm of the membranous ventricular septum. Br Heart J, 29: 60-63.

11. Major RH (1954) A history of medicine. Vol. 1. Charles C Thomas Publisher, Springfield, pp. 188-203, 488-497.

12. May MT (1968) Galen: on the usefulness of the parts of the body I-II. Cornell University Press Ithaca, New York, pp. 323-324, 347, 688-694.

13. Misra KP, Hildner FJ, Cohen LS, Narula OS, Samet P (1970) Aneurysm of the membranous ventricular septum: a mechanism for spontaneous closure of ventricular septal defect. N Engl J Med, 283: 58-61.

14. Perez Riera AR, Uchida AH, SchapachnikE, Dubner S, Zhang L, Filho CF, Ferreira C, Ferrara DE, de Luna AB, Moffa PJ (2008) The history of left septal fascicular block: chronological considerations of a reality yet to be universally accepted. Indian Pacing Electrophysiol J, 8: 114-128.

15. Perloff J K, Braunwald E (1997) Physical examination of the heart and circulation. In: Braunwald E ed. Heart disease. $5^{\text {th }}$ Ed. W B Saunders, Philadelphia, pp. 34-35.

16. Singer C (1956) Galen: on anatomical procedures. Oxford University Press, Oxford, pp. 2-5, 172-214.

17. Torrent-Guasp F, Buckberg GD, Clemente C, Cox JL, Coghlan CH, Gharib M (2001) The structure and function of the helical heart and its buttress wrapping. I. The normal macroscopic structure of the heart. Seminars Thor Cardiovasc Surg, 13: 301-319.

18. Williams PL, Warrick R, Dyson M, Bannister L ed. (1989) Grays's anatomy. $37^{\text {th }}$ Ed. Churchill Livingstone, Edinburg, London, Melbourne, New York, p. 793. 